\title{
Die Integrierte Versorgung ist die Medizin der Zukunft
}

\author{
Entgegen dem Eindruck, den viele Leserbriefe vermitteln, arbeitet schon heute mehr \\ als die Hälfte der Hausärzte in Managed-Care-Modellen, Tendenz steigend. Inte- \\ grierte Versorgung stärkt die Grundversorgung und hat das Potential zur Verbesse- \\ rung der wirtschaftlichen Situation der Hausärzte. Gewinner sind auch die Patienten \\ und Kostenträger.
}

Heinrich Zürcher

Mitglied der Geschäftsleitung der Argomed Ärzte AG
Viele Leserbriefe in der Schweizerischen Ärztezeitung (SÄZ) drücken eine Managed-Care-Aversion aus. Diese negativen Meinungen täuschen darüber hinweg, dass Managed Care bei den Hausärztinnen und Hausärzten bereits heute einen hohen Stellenwert hat. Über die Hälfte von ihnen engagiert sich freiwillig in Managed-Care-Modellen. Schon gegen 20\% der Versicherten vertrauen auf diese Versicherungsmodelle. Argomed setzt sich seit ihrer Gründung vor 12 Jahren für Managed Care ein. Unsere Erfahrungen sind durchwegs positiv, und die Aversionen beruhen unseres Erachtens weitgehend auf Vorurteilen und schlechter Information.

\section{Freiwillig und unabhängig}

Managed Care ist freiwillig, für Ärzte wie auch für Versicherte, und soll es auch bleiben. Auch in Managed-Care-Modellen können Patienten ihren Arzt

\section{Managed Care ist Qualitätsmedizin}

Wer in einem Hausärztenetz mitmacht, verpflichtet sich zur Teilnahme an einem Qualitätszirkel. Was als lästige Pflicht erscheint, stellt sich als lustvolle Tätigkeit heraus. Die medizinischen Themen, die sich der Qualitätszirkel selbst gibt, haben Priorität. Aus dem Gedankenaustausch («wie macht ihr das?») entwickelt sich ein Konsens bis hin zur Guideline. Ökonomische Themen sind von untergeordneter Priorität. Einen grossen Stellenwert hat die «Chropfleerete». Entwicklungen von der regionalen Gesundheitsversorgung bis hin zur kantonalen und schweizerischen Gesundheitspolitik werden diskutiert. Spezialisten erhalten die Möglichkeit, sich einzubringen und Zuweisungskriterien im Ärztenetz mitzugestalten. Wir machen uns eine eigene Meinung zu gesundheitspolitischen und standespolitischen Themen. Gutorganisierte Ärzte sind gutinformierte Ärzte!

\section{Der Datenaustausch zwischen den Vertragspartnern muss professionell organisiert werden, damit der Administrationsaufwand für uns Ärzte klein bleibt.}

Korrespondenz: Dr. med. Heinrich Zürcher Argomed Ärzte AG Täferstrasse 16 CH-5405 Dättwi

heinrich.zuercher@hin.ch wählen. Sie haben weiterhin ihren persönlichen Hausarzt, und sinnvolle Leistungen werden ihnen nicht vorenthalten. Sie verpflichten sich jedoch, mit wenigen Ausnahmen immer zuerst ihren Hausarzt aufzusuchen (Gatekeeping). Wer das nicht will, dem bleibt die Option, für den unkontrollierten Medizinkonsum voraussichtlich 500 bis 700 Franken pro Jahr zu bezahlen. Auch für die Hausärzte ist die Teilnahme freiwillig. Wer nicht von Managed Care überzeugt ist, kann abseits bleiben. Wer sich für die Teilnahme entscheidet, hat die Wahl zwischen HMOs und Hausarztmodellen. Mit der Ausnahme einiger weniger kasseneigener HMOs sind alle Ärztenetze selbstbestimmend und nicht Befehlsempfänger der Krankenversicherer.

\section{Gutorganisierte Hausärzte finden Gehör}

So basisdemokratisch die Auseinandersetzung über Leserbriefe in der SÄZ ist, bleibt sie doch auf individuelle Meinungsäusserungen beschränkt. Diese kontroverse Diskussion eignet sich nicht, um auf die Gesundheitspolitik Einfluss zu nehmen. Dafür brauchen wir tatkräftige Organisationen mit professionellen Ressourcen. Nur so können wir staatlichen Stellen, Versicherern und Spitälern auf Augenhöhe gegenübertreten. Dank Managed Care haben sich regionale Ärztenetze gebildet, die sich zu Betriebsgesellschaften zusammengetan haben. Dies hat auch die Standesorganisationen der Grundversorger gestärkt. Heute verfügen wir, ausgelöst durch Mana- 
ged Care, über Organisationen, welche die spezifischen Interessen der Hausärzte vertreten (notabene auch der Nicht-Managed-Care-Ärzte).

\section{Integrierte Versorgung ist die zeitgemässe Medizin}

Altbekannte Prinzipien wie Kollegialität, Wertschätzung und Respekt prägen die Zusammenarbeit unter den Medizinalpersonen. Unter ökonomischem Druck dürfen diese Prinzipien nicht leiden. Sie erlauben auch künftig einvernehmliche Vereinbarungen über die interdisziplinäre Zusammenarbeit. Jeder Akteur leistet die Arbeit, die in seine Kompetenz fällt. Die reibungslose Zusammenarbeit ist ein Gebot der Stunde und für unsere Patienten lebenswichtig. Die traditionelle, informelle Zusammenarbeit lässt viele Patienten durch Risse im Netz fallen. Die Zusammenarbeit entwickelt sich jetzt weiter $\mathrm{zu}$ einem strukturierten, sicheren und wohlorganisierten Behandlungsnetz mit Zusammenarbeitsvereinbarungen, die auch für die künftigen DRGs unverzichtbar sind.

\section{Die Rolle der ärzteeigenen Managed- Care-Organisationen}

Voraussetzung für die Integrierte Versorgung sind Verträge zwischen Versicherern und Ärztenetzen. Solche Verträge können nicht mehr von Vereinsvorständen im Milizsystem verhandelt werden. Auch der Datenaustausch zwischen den Vertragspartnern muss professionell organisiert werden, damit der Administrationsaufwand für uns Ärzte klein bleibt. Dies sind Aufgaben der ärzteeigenen Managed-Care-Organisationen, beispielsweise der Betriebsgesellschaften. Auch Mehrleistungen über die TARMED-Standards hinaus können angeboten werden und ausserhalb des Tarifs entlöhnt werden. In Zukunft werden Hausärzte einen Teil ihres Einkommens durch ihre Rolle in der Integrierten Medizin erzielen.

\section{Positive Signale an den Nachwuchs}

Lange genug haben wir über unsere schlechte Situation gejammert. Immerhin ist diese der Öffentlichkeit bewusst geworden, auch wenn sich an der wirtschaftlichen Situation noch nichts zum Positiven geändert hat. Als Negativeffekt haben wir den ärztlichen Nachwuchs abgeschreckt. Am Horizont zeichnen sich Hoffnungsschimmer ab (Gruppenpraxen,

\begin{abstract}
Argomed
Argomed ist die grösste ärztliche Managed-Care (MC)-Organisation mit 600 Ärzten in 18 Ärztenetzen in mehreren Kantonen, mit 200000 MCVersicherten. Das vielfältige Dienstleistungsangebot ist gewachsen aus dem MC-Verhandlungssupport und der MC-Abwicklung mit Auswertung der MC-Daten. Die Qualitätszirkel und die Ärztenetze bekommen logistische Unterstützung. Innovative Zusatzangebote über TARMED hinaus werden entwickelt, mit entsprechender Entschädigung.

Ein neues Angebot ist der MehrFachArzt, das einzige Qualitätslabel, das nur für Hausärzte entwickelt wurde. MehrFachArzt ist das Zertifikat für die wirklich relevanten Qualitätskriterien von Hausärzten. Weitere Aktivitäten zur Unterstützung der Hausärzte sind die Förderung von eHealth mit der elektronischen Krankengeschichte und dem elektronischen Datenaustausch. Mit Spitälern und Spezialärztegruppen werden Zusammenarbeitsvereinbarungen abgeschlossen. CIRS ist das Meldesystem für kritische Ereignisse. Das Projekt KIMSA zur Meldung potentiell langwieriger Heilungsverläufe wurde mit der SUVA zusammen entwickelt. Neu bietet Argomed auch Unterstützung beim Aufbau und Betrieb von Gruppenpraxen.
\end{abstract}

Teilzeittätigkeit, Entlöhnung für Tätigkeiten im Rahmen der Integrierten Versorgung). Die Integrierte Versorgung bietet Chancen zur Verbesserung unserer wirtschaftlichen Situation.

\section{Fazit}

Mehr als die Hälfte der Grundversorger ist schon heute freiwillig bei Managed Care dabei. Die Teilnahme erlaubt die Mitgliedschaft in einer engagierten Ärztegruppe und bringt jeder Hausärztin und jedem Hausarzt und auch ihren Patientinnen und Patienten direkten Nutzen. Die Hausärztebewegung ist stark und progressiv. Professionelle Strukturen erhalten den Schwung und stärken den Einfluss im Gesundheitswesen. Dieses positive Klima wird dazu führen, das letzte Eis der Managed-Care-Aversion zu schmelzen. 